\title{
Performance Analysis for Distributed Classification Fusion Using Soft-Decision Decoding in Wireless Sensor Networks
}

\author{
Jing-Tian Sung ${ }^{1}$, Hung-Ta Pai ${ }^{2}$, and Bih-Hwang Lee ${ }^{3}$ \\ ${ }^{1}$ Dept. of Electrical Engineering \\ National Taiwan University of Science and Technology \\ No. 43, Sec. 4, Keelung Rd., Taipei, 106 Taiwan \\ d9107305@mail.ntust.edu.tw \\ ${ }^{2}$ Graduate Institute of Communication Engineering \\ National Taipei University \\ No. 151, University Rd., Sanhsia, Taipei, 237 Taiwan \\ htpai@mail.ntpu.edu.tw \\ ${ }^{3}$ Dept. of Electrical Engineering \\ National Taiwan University of Science and Technology \\ No. 43, Sec. 4, Keelung Rd., Taipei, 106 Taiwan \\ lee@ccg.ee.ntust.edu.tw
}

\begin{abstract}
Distributed Classification Fusion using Error-Correcting Codes (DCFECC) has recently been proposed for wireless sensor networks. It adopts the Minimum Hamming Distance (MHD) fusion rule and performs much better than traditional classification approaches when the network has faulty sensors. Different fusion rules were proposed later. One of them is Distributed Classification fusion using Soft-decision Decoding (DCSD). The DCSD fusion rule has a considerably lower misclassification probability than the MHD fusion rule. This work analyzes the performance of the DCSD fusion rule. Asymptotic performance approximation of the DCSD fusion rule is derived based on the Central Limit Theorem. Furthermore, an asymptotic upper bound on the misclassification probability is obtained. Finally, numerical simulations are conducted to verify our analysis results.
\end{abstract}

Keywords: Wireless sensor networks, distributed detection, softdecision decoding, Central Limit Theorem.

\section{Introduction}

Wireless sensor networks (WSNs) comprise many tiny, low-cost, battery-powered sensors in a small area. The sensors detect environmental variations and then transmit the detection results to other sensors or a base station. The base station or a sensor, serving as a fusion center, collects all detection results, and determines what phenomenon has occurred [1,2]. The WSN sometimes must be able to function under severe conditions, such as in a battlefield, fireplace or polluted area. The transmission channel, as well as the environmental phenomenon 
observed by the sensor, is noisy. Furthermore, the observation signal to noise ratio (OSNR) and the channel signal to noise ratio (CSNR) may change quickly and be difficult to estimate accurately. Some sensors may even have unrecognized faults in the harsh environment. Therefore, a fault-tolerant system must be developed to make the received local decisions error-resistant [3, 4.

Wang et al. [5] proposed Distributed Classification Fusion using ErrorCorrecting Codes (DCFECC) to solve this problem by combining the distributed detection theory [6] with the concept of error-correcting codes in communication systems 7. DCFECC with the Minimum Hamming Distance (MHD) fusion rule has a much lower probability of misclassification when some sensors are faulty than the traditional distributed classification method. DCFECC outperforms the method even when CSNR is not correctly estimated. Its performance analysis is given in 8 .

Three fusion rules were proposed and compared [9, 10, later. One is the maximum a posteriori probability (MAP) fusion rule, one is the Minimum Euclean Distance (MED) fusion rule, and the other is Distributed Classification fusion using Soft-decision Decoding (DCSD) fusion rule. The MAP and DCSD fusion rules have a considerably misclassification probability than the MED one. Moreover, the DCSD has a lower computational complexity than the MAP with little performance loss when no faulty sensor appears. If some sensors are defective, the DCSD outperforms the MAP when the misclassification probability is lower than 0.2 . Therefore, the DCSD fusion rule is a more practical choice than the other ones. However, its performance analysis have not been provided.

In this work, we analyze the performance of the DCSD fusion rule without assuming no errors in local decisions and wireless channels. Asymptotic performance approximations are obtained by the Central Limit Theorem. Asymptotic upper bounds on the misclassification probability are derived. These results can be utilized for the optimal code matrix design in the future. Computer simulations show the performance approximation is accurate and the upper bound is tight when the misclassification probability is lower than 0.2 .

The remainder of this work is organized as follows. Section 2 briefly addresses the distributed detection problem in WSNs and the DCSD fusion rule. The performance analysis of the DCSD fusion rule is derived in Section 3 . Section 4 shows simulation results. Concluding remarks and suggestions for future works are given in Section 5 .

\section{Fault-Tolerant Distributed Detection and DCSD Fusion Rule}

Figure 1 depicts a wireless sensor network for distributed detection with $N$ sensors deployed for collecting environment variation data and a fusion center for making a final decision of detections. At the $j$ th sensor, one observation $y_{j}$ is undertaken for one of phenomena $H_{i}$, where $i=1,2, \ldots, M$. The observation is normally a real number represented by many bits. Transmitting the real number to the fusion center would consume too much power, so a local decision, $u_{j}$, is 




Fig. 1. Structure of a wireless sensor network for distributed detection using $N$ sensors

made instead. For a phenomenon, if only $L$ bits are allowed to send the local decision from the sensor to the fusion center, then the $L$ bits are used to represent the decision.

The DCFECC approach [5] sets $L=1$, and designs an $M \times N$ code matrix $\mathbf{T}$ not only to correct transmission errors, but also to resist faulty sensors. The application of the code matrix is derived from error-correcting codes. Table 11ists an example of $\mathbf{T}$, which is the optimal code matrix found through the criterion in [11. Row $i$ of the matrix represents a codeword $\mathbf{c}_{i}=\left(c_{i, 1}, c_{i, 2}, \ldots, c_{i, N}\right)$ corresponding to hypothesis $H_{i}$, and $c_{i, j}$ denotes a 1-bit symbol corresponding to the decision of sensor $j$. Notably, sensors 1 to 10 have the same decision pattern and sensors 11 to 20 have the same decision pattern. As a result, there are two decision patterns for the code matrix in Table 1

Table 1 . The $4 \times 20$ optimal code matrix

\begin{tabular}{l|llllllllllllllllllll}
\hline$H_{1}$ & 1 & 1 & 1 & 1 & 1 & 1 & 1 & 1 & 1 & 1 & 0 & 0 & 0 & 0 & 0 & 0 & 0 & 0 & 0 & 0 \\
$H_{2}$ & 1 & 1 & 1 & 1 & 1 & 1 & 1 & 1 & 1 & 1 & 1 & 1 & 1 & 1 & 1 & 1 & 1 & 1 & 1 & 1 \\
$H_{3}$ & 0 & 0 & 0 & 0 & 0 & 0 & 0 & 0 & 0 & 0 & 1 & 1 & 1 & 1 & 1 & 1 & 1 & 1 & 1 & 1 \\
$H_{4}$ & 0 & 0 & 0 & 0 & 0 & 0 & 0 & 0 & 0 & 0 & 0 & 0 & 0 & 0 & 0 & 0 & 0 & 0 & 0 & 0 \\
\hline
\end{tabular}

Let $v_{j}$ be the received local decision at the fusion center, where $v_{j} \in\{0,1\}$. A cost function is then defined as

$$
C_{\mathbf{v}, \mathbf{c}_{i}}= \begin{cases}1-\frac{1}{q}, & \mathbf{c}_{i} \text { is one of } q \text { solutions of } \\ & \arg \min _{\mathbf{c}_{k}} d_{H}\left(\mathbf{v}, \mathbf{c}_{k}\right) \\ 1, \quad & \text { else. }\end{cases}
$$


Notably, $d_{H}\left(\mathbf{v}, \mathbf{c}_{k}\right)$ denotes the Hamming distance between a received vector, $\mathbf{v}=\left(v_{1}, v_{2}, \ldots, v_{N}\right)$, and a codeword, $\mathbf{c}_{k}$. Hence, the Bayes risk function [6] represents the probability of misclassification,

$$
P_{e}=\sum_{i, \mathbf{v}} \int_{\mathbf{y}} p\left(\mathbf{v}, \mathbf{y}, H_{i}\right) C_{\mathbf{v}, \mathbf{c}_{i}} d \mathbf{y},
$$

where $\mathbf{y}=\left(y_{1}, y_{2}, \ldots, y_{N}\right)$. Set $\mathbf{u}=\left(u_{1}, u_{2}, \ldots, u_{N}\right)$, and make the following assumptions:

Assumption 1: Observations at all sensors are conditionally independent, i.e.,

$$
p\left(\mathbf{y} \mid H_{i}\right)=p\left(y_{1}, y_{2}, \ldots, y_{N} \mid H_{i}\right)=\prod_{j=1}^{N} p\left(y_{j} \mid H_{i}\right) .
$$

Assumption 2: The $j$ th local decision, $u_{j}$, only depends on the $j$ th observation, $y_{j}$.

Assumption 3: The $j$ th received local decision, $v_{j}$, only depends on the $j$ th local decision, $u_{j}$.

Equation (1) can then be recast as

$$
\begin{aligned}
P_{e}= & \sum_{i, \mathbf{u}, \mathbf{v}-v_{j}} \int_{\mathbf{y}} P\left(H_{i}\right)\left[P\left(\mathbf{v}_{j=1} \mid \mathbf{u}\right) p(\mathbf{u} \mid \mathbf{y}) p\left(\mathbf{y} \mid H_{i}\right) C_{\mathbf{v}_{j=1}, \mathbf{c}_{i}}\right. \\
& \left.+P\left(\mathbf{v}_{j=0} \mid \mathbf{u}\right) p(\mathbf{u} \mid \mathbf{y}) p\left(\mathbf{y} \mid H_{i}\right) C_{\mathbf{v}_{j=0}, \mathbf{c}_{i}}\right] d \mathbf{y}
\end{aligned}
$$

where $\mathbf{v}_{j=b_{v}}=\left(v_{1}, \ldots, v_{j-1}, b_{v}, v_{j+1}, \ldots, v_{N}\right), b_{v} \in\{0,1\}$, and $\mathbf{v}-v_{j}$ represents the elements of $\mathbf{v}$ except $v_{j}$.

The DCSD is applied as follows. Set $\mathbf{u}=\left(u_{1}, u_{2}, \ldots, u_{N}\right)$. The local decision $\mathbf{u}$ is transmitted for the final decision to the fusion center. The received data at the fusion center are $\tilde{\mathbf{v}}=\left(\tilde{v}_{1}, \tilde{v}_{2}, \ldots, \tilde{v}_{N}\right)$, where

$$
\tilde{v}_{j}=(-1)^{u_{j}} \sqrt{\frac{E_{s}}{L}}+n_{j} .
$$

Notice that $E_{s}$ is the total transmission energy per sensor, and $n_{j}$ is the additive white Gaussian noise (AWGN) with the two-sided power spectral density $N_{0} / 2$. The received data are decoded as hypothesis $i$ if

$$
p\left(\tilde{\mathbf{v}} \mid \mathbf{c}_{i}\right) \geq p\left(\tilde{\mathbf{v}} \mid \mathbf{c}_{k}\right) \text { for all } \mathbf{c}_{k}, \text { where } k=1, \ldots, M .
$$

For simplicity, let $L=1$. Since $\tilde{v}_{j}$ does not depend on $c_{i, j}$ given $u_{j}$, and according to Assumptions 2 and 3, (3) can be rewritten as

$$
\begin{gathered}
\prod_{j=1}^{N} \sum_{b_{u}=0}^{1} p\left(\tilde{v}_{j} \mid u_{j}=b_{u}\right) p\left(u_{j}=b_{u} \mid c_{i, j}\right) \geq \prod_{j=1}^{N} \sum_{b_{u}=0}^{1} p\left(\tilde{v}_{j} \mid u_{j}=b_{u}\right) p\left(u_{j}=b_{u} \mid c_{k, j}\right), \\
\Rightarrow \sum_{j=1}^{N} \ln \frac{\sum_{b_{u}=0}^{1} p\left(\tilde{v}_{j} \mid u_{j}=b_{u}\right) p\left(u_{j}=b_{u} \mid c_{i, j}\right)}{\sum_{b_{u}=0}^{1} p\left(\tilde{v}_{j} \mid u_{j}=b_{u}\right) p\left(u_{j}=b_{u} \mid c_{k, j}\right)} \geq 0
\end{gathered}
$$


Because $c_{i, j}$ and $c_{k, j}$ are binary, the bit logarithm-likelihood ratio of the received data at the fusion center can be defined as

$$
\lambda_{j}=\ln \frac{\sum_{b_{u}=0}^{1} p\left(\tilde{v}_{j} \mid u_{j}=b_{u}\right) p\left(u_{j}=b_{u} \mid c_{i, j}=0\right)}{\sum_{b_{u}=0}^{1} p\left(\tilde{v}_{j} \mid u_{j}=b_{u}\right) p\left(u_{j}=b_{u} \mid c_{k, j}=1\right)} .
$$

(44) is then equivalent to

$$
\sum_{j=1}^{N}\left[\lambda_{j}-(-1)^{c_{i, j}}\right]^{2} \leq \sum_{j=1}^{N}\left[\lambda_{j}-(-1)^{c_{k, j}}\right]^{2} .
$$

\section{Performance Analysis}

Assume that the wireless channel between the fusion center and the sensor is influenced by AWGN with zero mean and variance $\sigma_{c}^{2}$. Namely,

$$
p\left(\tilde{v}_{j} \mid u_{j}=b_{u}\right)=\frac{1}{\sqrt{2 \pi \sigma_{c}^{2}}} \exp \left\{-\frac{\left(\tilde{v}_{j}-(-1)^{b_{u}}\right)^{2}}{2 \sigma_{c}^{2}}\right\} .
$$

For simplicity, let

$$
\begin{aligned}
& P_{j, 0 \mid 0}=p\left(u_{j}=0 \mid c_{k, j}=0\right) \\
& P_{j, 1 \mid 1}=p\left(u_{j}=1 \mid c_{k, j}=1\right) .
\end{aligned}
$$

Substituting (7) and (8) into (5), we can rewritte logarithm-likelihood ratio as

$$
\begin{aligned}
\lambda_{j} & =\ln \frac{\exp \left\{\frac{\tilde{v}_{j}}{\sigma_{c}^{2}}\right\} P_{j, 0 \mid 0}+\exp \left\{-\frac{\tilde{v}_{j}}{\sigma_{c}^{2}}\right\}\left(1-P_{j, 0 \mid 0}\right)}{\exp \left\{\frac{\tilde{v}_{j}}{\sigma_{c}^{2}}\right\}\left(1-P_{j, 1 \mid 1}\right)+\exp \left\{-\frac{\tilde{v}_{j}}{\sigma_{c}^{2}}\right\} P_{j, 1 \mid 1}} \\
= & \ln \frac{\exp \left\{\frac{2 \tilde{v}_{j}}{\sigma_{c}^{2}}\right\} P_{j, 0 \mid 0}+\left(1-P_{j, 0 \mid 0}\right)}{\exp \left\{\frac{2 \tilde{v}_{j}}{\sigma_{c}^{2}}\right\}\left(1-P_{j, 1 \mid 1}\right)+P_{j, 1 \mid 1}} .
\end{aligned}
$$

Thus, the Cumulative Density Function (CDF) of $\lambda_{j}$ can be expressed as

$$
\begin{aligned}
\operatorname{Pr}\left(\lambda_{j}<x \mid c_{i, j}\right) & =\operatorname{Pr}\left\{\ln \frac{\exp \left\{\frac{2 \tilde{v}_{j}}{\sigma_{c}^{2}}\right\} P_{j, 0 \mid 0}+\left(1-P_{j, 0 \mid 0}\right)}{\exp \left\{\frac{2 \tilde{v}_{j}}{\sigma_{c}^{2}}\right\}\left(1-P_{j, 1 \mid 1}\right)+P_{j, 1 \mid 1}}<x \mid c_{i, j}\right\} \\
& =\operatorname{Pr}\left\{\tilde{v}_{j}<\frac{\sigma_{c}^{2}}{2} \ln \frac{e^{x} P_{j, 1 \mid 1}+P_{j, 0 \mid 0}-1}{P_{j, 0 \mid 0}+e^{x}\left(P_{j, 1 \mid 1}-1\right)} \mid c_{i, j}\right\} .
\end{aligned}
$$


We denote

$$
\zeta_{j}(x)=\frac{\sigma_{c}^{2}}{2} \ln \left(\frac{e^{x} P_{j, 1 \mid 1}+P_{j, 0 \mid 0}-1}{P_{j, 0 \mid 0}+e^{x}\left(P_{j, 1 \mid 1}-1\right)}\right) .
$$

Because the Probability Density Function $(\mathrm{PDF})$ of $\tilde{v}_{j}$ can be represented by

$$
\begin{aligned}
f_{\tilde{v}_{j}}\left(x \mid c_{i, j}\right)= & \frac{P_{j, c_{i, j} \mid c_{i, j}}}{\sqrt{2 \pi \sigma_{c}^{2}}} \exp \left\{-\frac{x-(-1)^{c_{i, j}}}{2 \sigma_{c}^{2}}\right\} \\
& +\frac{\left(1-P_{j, c_{i, j} \mid c_{i, j}}\right)}{\sqrt{2 \pi \sigma_{c}^{2}}} \exp \left\{-\frac{x-(-1)^{\left(1-c_{i, j}\right)}}{2 \sigma_{c}^{2}}\right\},
\end{aligned}
$$

where $P_{j, c_{i, j} \mid c_{i, j}}$ represents the probability of correct local decision for the sensor $j$, 10. can be rewritten as

$$
\begin{aligned}
& \operatorname{Pr}\left(\tilde{v}_{j}<\zeta_{j}(x) \mid c_{i, j}\right) \\
& =\int_{-\infty}^{\zeta_{j}(x)} f_{\tilde{v}_{j}}\left(x \mid c_{i, j}\right) d x \\
& =P_{j, c_{i, j} \mid c_{i, j}} \times \Phi\left(\frac{\zeta_{j}(x)-(-1)^{c_{i, j}}}{\sigma_{c}}\right) \\
& \quad+\left(1-P_{j, c_{i, j} \mid c_{i, j}}\right) \times \Phi\left(\frac{\zeta_{j}(x)-(-1)^{c_{i, j}}}{\sigma_{c}}\right),
\end{aligned}
$$

where $\Phi(\cdot)$ is the CDF of a random variable with normal distribution, i.e.,

$$
\Phi(x)=\frac{1}{\sqrt{2 \pi}} \int_{-\infty}^{x} \exp \left\{-\frac{x^{2}}{2}\right\} d x .
$$

Therefore, the PDF of $\lambda_{j}$ can be given by

$$
\begin{aligned}
& f_{\lambda_{j}}\left(x \mid c_{i, j}\right)=\frac{d}{d x} \int_{-\infty}^{\zeta_{j}(x)} f_{\tilde{v}_{j}}\left(t \mid c_{i, j}\right) d t \\
& =\frac{1}{2}\left[\frac{P_{j, c_{i, j} \mid c_{i, j}}}{\sqrt{2 \pi \sigma_{c}^{2}}} \exp \left\{-\frac{\zeta(x)-(-1)^{c_{i, j}}}{2 \sigma_{c}^{2}}\right\}\right. \\
& \left.+\frac{\left(1-P_{j, c_{i, j} \mid c_{i, j}}\right)}{\sqrt{2 \pi \sigma_{c}^{2}}} \exp \left\{-\frac{\zeta(x)-(-1)^{\left(1-c_{i, j}\right)}}{2 \sigma_{c}^{2}}\right\}\right] \\
& \quad \times \frac{\sigma_{c}^{2} e^{x}\left(P_{j, 1 \mid 1}+P_{j, 0 \mid 0}\right)}{\left(e^{x} P_{j, 1 \mid 1}+P_{j, 0 \mid 0}-1\right)\left(e^{x} P_{j, 1 \mid 1}+P_{j 0 \mid 0}-e^{x}\right)}
\end{aligned}
$$

The mean and the variance of $\lambda_{j}$ can be found as

$$
\mu_{\lambda_{j}}=\int_{-\infty}^{\infty} x f_{\lambda_{j}}\left(x \mid c_{i, j}\right) d x
$$




$$
\sigma_{\lambda_{j}}^{2}=\int_{-\infty}^{\infty}\left(x-\mu_{\lambda_{j}}\right)^{2} f_{\lambda_{j}}\left(x \mid c_{i, j}\right) d x
$$

respectively.

The misclassification probability at the fusion center can be expressed by

$$
P_{e}=\sum_{i=1}^{M} \operatorname{Pr}\left(H_{i}\right) \operatorname{Pr}\left(\bigcup_{k=1, k \neq i}^{M} \tilde{H}_{k} \mid H_{i}\right) .
$$

When the DCSD fusion rule is employed, the misclassification probability given that $H_{i}$ occurs can be derived as follows.

$$
\begin{aligned}
\operatorname{Pr}\left(\tilde{H}_{k} \mid H_{i}\right) & =\operatorname{Pr}\left(\sum_{j=1}^{N}\left[\lambda_{j}-(-1)^{c_{i, j}}\right]^{2} \geq \sum_{j=1}^{N}\left[\lambda_{j}-(-1)^{c_{k, j}}\right]^{2} \mid H_{i}\right) \\
& =\operatorname{Pr}\left(\sum_{j=1}^{N} \lambda_{j}\left(c_{k, j}-c_{i, j}\right) \leq 0 \mid H_{i}\right),
\end{aligned}
$$

where $k \neq i$. Let $S$ be the number of the decision patterns for a code matrix and $\Omega(\ell), \ell=1,2, \ldots, S$, be the set of sensors with the same decision pattern $\ell$. For example, $\Omega(1)=\{1,2, . .10\}$ and $\Omega(2)=\{11,12, . .20\}$ for the code matrix in Table 1. Since the sensors with the same decision pattern operate identically, the means and the variances of $\lambda_{j}$, where $j \in \Omega(\ell)$, have no difference and can be denoted as $\mu_{\ell}$ and $\sigma_{\ell}^{2}$, respectively. Moreover, define $d_{H}^{(\ell)}\left(\mathbf{c}_{k}, \mathbf{c}_{i}\right)$ as the partial Hamming distance between $\mathbf{c}_{k}$ and $\mathbf{c}_{i}$ at the set $\ell$. For example, $d_{H}^{(1)}\left(\mathbf{c}_{1}, \mathbf{c}_{2}\right)=0$ and $d_{H}^{(2)}\left(\mathbf{c}_{1}, \mathbf{c}_{3}\right)=10$ for the code matrix in Table 11. The sensor sets $\Omega(\ell)$ satisfying $d_{H}^{(\ell)}\left(\mathbf{c}_{i}, \mathbf{c}_{k}\right) \neq 0$ are employed to differentiate $H_{k}$ from $H_{i}$ at the fusion center. When the information from two or more sensor sets are utilized for the final decision, the Hamming distance between $\mathbf{c}_{i}$ and $\mathbf{c}_{k}$ is large. Because of the large Hamming distance, the probability of misclassification is small. Therefore, when $\left\|\left\{\ell: d_{H}^{(\ell)}\left(\mathbf{c}_{i}, \mathbf{c}_{k}\right) \neq 0\right\}\right\| \geq 2$, (18) can be rewritten and approximated as

$$
\begin{aligned}
\operatorname{Pr}\left(\tilde{H}_{k} \mid H_{i}\right) & =\operatorname{Pr}\left(\sum_{\left\{\ell: d_{H}^{(\ell)}\left(\mathbf{c}_{i}, \mathbf{c}_{k}\right) \neq 0\right\}} \sum_{j \in \Omega(\ell)} \lambda_{j}\left(c_{k, j}-c_{i, j}\right) \leq 0 \mid H_{i}\right) \\
& \approx \prod_{\left\{\ell: d_{H}^{(\ell)}\left(\mathbf{c}_{i}, \mathbf{c}_{k}\right) \neq 0\right\}} \operatorname{Pr}\left(\sum_{j \in \Omega(\ell)} \lambda_{j}\left(c_{k, j}-c_{i, j}\right) \leq 0 \mid H_{i}\right) .
\end{aligned}
$$

The following corollary can be obtained based on the Central Limit Theorem. 
Corollary 1. If $d_{H}^{(\ell)}\left(\mathbf{c}_{k}, \mathbf{c}_{i}\right)$ is sufficiently large, the misclassification probability can be approximated as

$$
\operatorname{Pr}\left(\tilde{H}_{k} \mid H_{i}\right) \approx \prod_{\left\{\ell: d_{H}^{(\ell)}\left(\mathbf{c}_{i}, \mathbf{c}_{k}\right) \neq 0\right\}} \Phi\left(-\frac{\sqrt{d_{H}^{(\ell)}\left(\mathbf{c}_{k}, \mathbf{c}_{i}\right)} \times\left(c_{k, j}-c_{i, j}\right) \mu_{\ell}}{\sigma_{\ell}}\right) .
$$

If the size of the code matrix is large, it is difficult to calculate the approximation according to (20). Since the probability of the union in (17) can be approximated as

$$
\sum_{k=1, k \neq i}^{M} \operatorname{Pr}\left(\tilde{H}_{k} \mid H_{i}\right)
$$

we can obtain the following approximation.

Corollary 2. If $d_{H}^{(\ell)}\left(\mathbf{c}_{k}, \mathbf{c}_{i}\right)$ is sufficiently large, the misclassification probability can be approximated as

$$
\begin{aligned}
& P_{e} \approx \sum_{i=1}^{M} \sum_{k=1, k \neq i}^{M} \operatorname{Pr}\left(H_{j}\right) \times \\
& \prod_{\left\{\ell: d_{H}^{(\ell)}\left(\mathbf{c}_{i}, \mathbf{c}_{k}\right) \neq 0\right\}} \Phi\left(-\frac{\sqrt{d_{H}^{(\ell)}\left(\mathbf{c}_{k}, \mathbf{c}_{i}\right)} \times\left(c_{k, j}-c_{i, j}\right) \mu_{\ell}}{\sigma_{\ell}}\right) .
\end{aligned}
$$

Define

$$
\begin{aligned}
& P_{e}^{*}=\sum_{i=1}^{M} \sum_{k=1, k \neq i}^{M} \operatorname{Pr}\left(H_{j}\right) \times \\
& \prod_{\left\{\ell: d_{H}^{(\ell)}\left(\mathbf{c}_{i}, \mathbf{c}_{k}\right) \neq 0\right\}} \Phi\left(-\frac{\sqrt{d_{H}^{(\ell)}\left(\mathbf{c}_{k}, \mathbf{c}_{i}\right)} \times\left(c_{k, j}-c_{i, j}\right) \mu_{\ell}}{\sigma_{\ell}}\right) .
\end{aligned}
$$

Next, we propose a corollary to derive the upper bound of the approximation.

Corollary 3. For all $\ell$ and all pair $\{i, k\}$, if

$$
\Phi\left(-\frac{\sqrt{d_{H}^{(\ell)}\left(\mathbf{c}_{k}, \mathbf{c}_{i}\right)} \times\left(c_{k, j}-c_{i, j}\right) \mu_{\ell}}{\sigma_{\ell}}\right) \leq \operatorname{Pr}\left(\sum_{j \in \Omega(\ell)}\left(c_{k, j}-c_{i, j}\right) \tilde{v}_{j} \leq 0\right)
$$

then

$$
P_{e}^{*} \leq \sum_{i=1}^{M} \sum_{k=1, k \neq i}^{M} \operatorname{Pr}\left(H_{i}\right) \times \prod_{\substack{\left\{\ell: d_{H}^{(\ell)} \\\left(\mathbf{c}_{i}, \mathbf{c}_{k}\right) \neq 0\right\}}} \operatorname{Pr}\left(\sum_{j \in \Omega(\ell)}\left(c_{k, j}-c_{i, j}\right) \tilde{v}_{j} \leq 0\right) .
$$


From (12), the characteristic function of $\tilde{v}_{j}$ is

$$
\begin{aligned}
\varphi(z)=P_{j, c_{i, j} \mid c_{i, j}} \exp \left\{\mathbf{j}\left(c_{k, j}-c_{i, j}\right) z-\frac{\sigma_{c}^{2} z^{2}}{2}\right\} \\
+\left(1-P_{j, c_{i, j} \mid c_{i, j}}\right) \exp \left\{-\mathbf{j}\left(c_{k, j}-c_{i, j}\right) z-\frac{\sigma_{c}^{2} z^{2}}{2}\right\}
\end{aligned}
$$

and the characteristic function of a random variable which is the summation of $\tilde{v}_{j}, j=1,2, \ldots, n$, is

$$
\begin{aligned}
\varphi^{n}(z)=\sum_{t=0}^{n}\left(\begin{array}{l}
n \\
t
\end{array}\right)\left(P_{j, c_{i, j} \mid c_{i, j}}\right)^{t}\left(1-P_{j, c_{i, j} \mid c_{i, j}}\right)^{(n-t)} \\
\quad \times \exp \left\{\mathbf{j}(2 t-n)\left(c_{k, j}-c_{i, j}\right)-\frac{z^{2}}{2}\left(n \sigma_{c}^{2}\right)\right\},
\end{aligned}
$$

where $\mathbf{j}=\sqrt{-1}$. Then, the PDF of a random variable which is the summation of $\tilde{v}_{j}, j=1,2, \ldots, n$, is

$$
\begin{aligned}
f_{\sum \tilde{v}_{j}}(x)= & \frac{1}{\sqrt{2 \pi n \sigma_{c}^{2}}} \sum_{t=0}^{n}\left(\begin{array}{l}
n \\
t
\end{array}\right)\left(P_{j, c_{i, j} \mid c_{i, j}}\right)^{t}\left(1-P_{j, c_{i, j} \mid c_{i, j}}\right)^{(n-t)} \\
& \times \exp \left\{-\frac{\left(x-(2 t-n)\left(c_{k, j}-c_{i, j}\right)\right)^{2}}{2 n \sigma_{c}^{2}}\right\} .
\end{aligned}
$$

Let $w_{\ell}=d_{H}^{(\ell)}\left(\mathbf{c}_{k}, \mathbf{c}_{i}\right)$. According to Corollary 3, when (22) holds, the upper bound can be expressed as

$$
\begin{aligned}
P_{e}^{*} \leq \sum_{i=1}^{M} \sum_{k=1, k \neq i}^{M} \operatorname{Pr}\left(H_{i}\right) \prod_{\left\{\ell: w_{\ell} \neq 0\right\}} \sum_{t=0}^{w_{\ell}}\left(\begin{array}{c}
w_{\ell} \\
t
\end{array}\right)\left(P_{j, c_{i, j} \mid c_{i, j}}\right)^{t} \\
\quad \times\left(1-P_{j, c_{i, j} \mid c_{i, j}}\right)^{w_{\ell}-t} \Phi\left(-\frac{\left(2 t-w_{\ell}\right)\left(c_{k, j}-c_{i, j}\right)}{\sqrt{w_{\ell}} \sigma_{c}}\right) .
\end{aligned}
$$

\section{Numerical and Simulation Results}

The proposed approximations and the upper bound are verified by simulations with $10^{6}$ Monte Carlo tests. A fusion center and $N=20$ sensors are deployed to detect and classify four hypotheses $H_{1}, H_{2}, H_{3}$ and $H_{4}$. We also assume that the local observations are interfered by the Gaussian noise with the same standard deviation $\sigma_{o}$ and mean $0,1,2$, and 3, respectively. In addition, wireless channels are interfered by AWGN and CSNR is $10 \times \log _{10}\left(E_{s} / N_{0}\right)$. The code matrix in Table 1 was utilized.

The first and second approximations are stated in Corollary 1 and Corollary2. respectively. The first set of figures shows the approximations and the simulation 


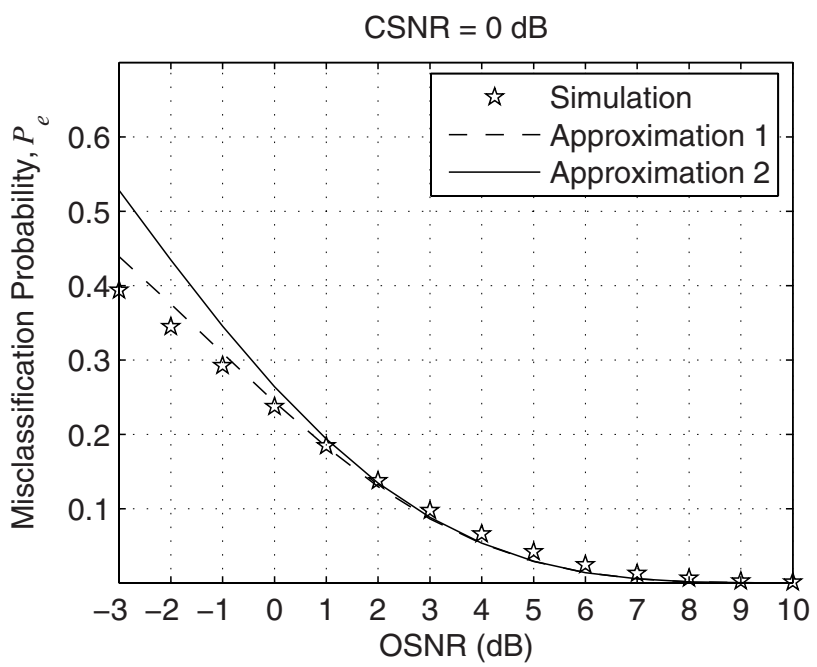

Fig. 2. Proposed approximations and simulation results when CSNR $=0 \mathrm{~dB}$

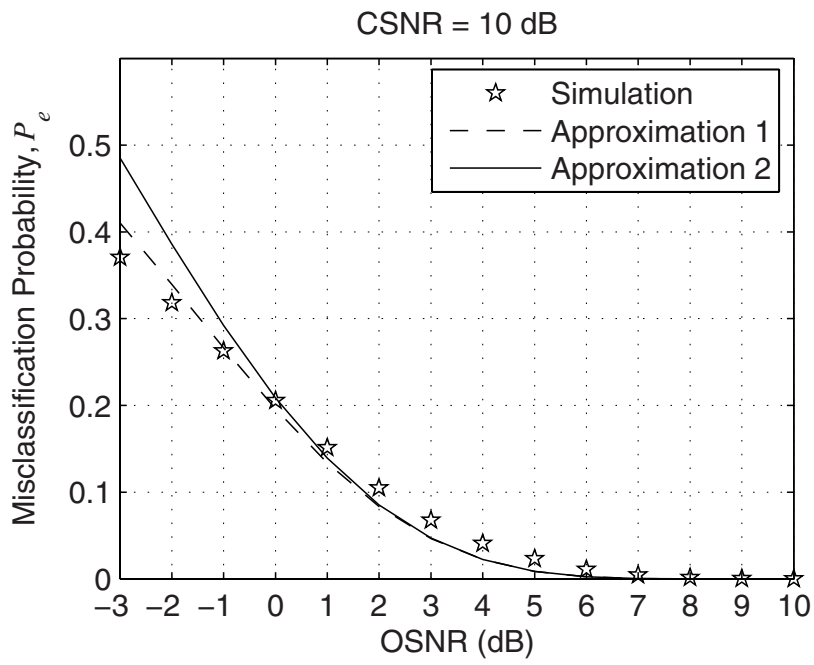

Fig. 3. Proposed approximations and simulation results when $\mathrm{CSNR}=10 \mathrm{~dB}$

result when CSNR is set to be 0 and 10, respectively. In this case, $M$ is small and the probability of the union in (17) is obtainable. As shown in Fig. 2 and 3 , both approximations are accurate when the misclassification probability is lower than 0.2 . The first approximation is better than the second one. However, the computational complexity of the first approximation is higher than the second one, as we pointed in the previous section. When OSNR is low, the probability of 


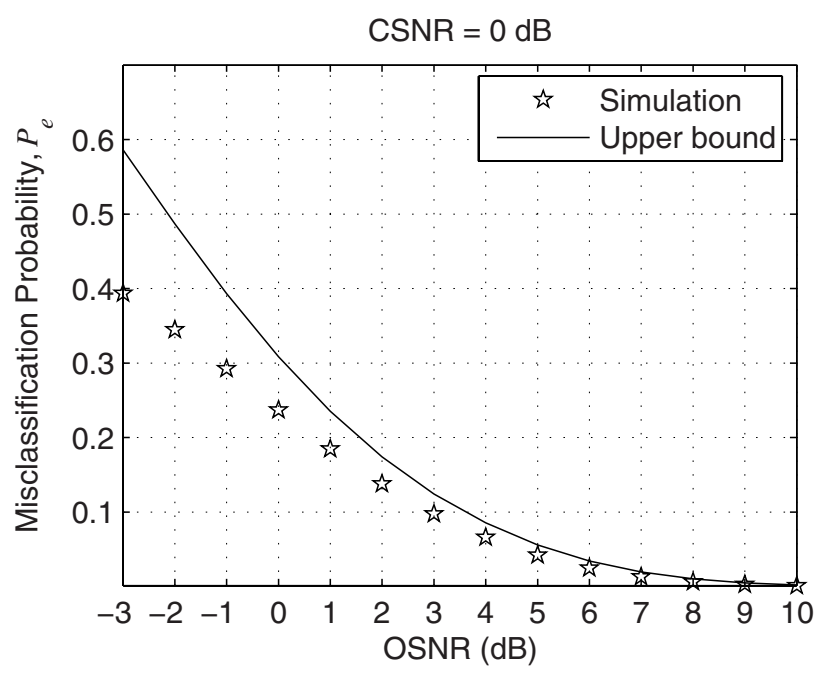

Fig. 4. Proposed upper bounds and simulation results when $\mathrm{CSNR}=0 \mathrm{~dB}$

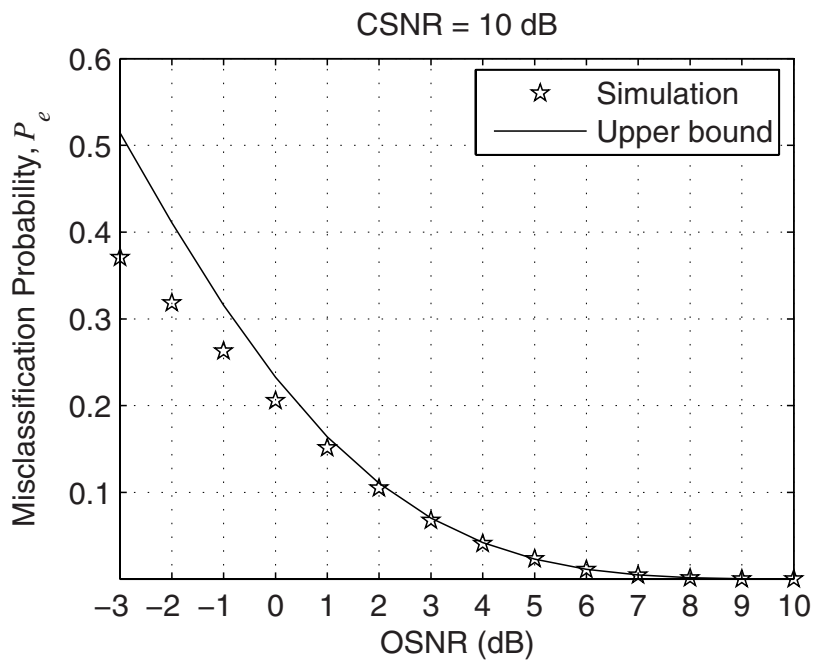

Fig. 5. Proposed upper bounds and simulation results when CSNR $=10 \mathrm{~dB}$

the union in (17) cannot be approximated by (21). Thus, the difference between the approximation and the simulation result is large at $-3 \mathrm{~dB}$. Figures 4 and 5 show that the upper bound in (27) is very close to the simulation result when the misclassification probability is lower than 0.2 . 


\section{Conclusions and Future Works}

This work analyzes the performance of the distributed detection using the DCSD fusion rule. Two approximations and an upper bound of the misclassification probability are presented. The analysis is based on the Central Limit Theorem. The simulation results showed that the approximation and the upper bound are accurate for the network with only twenty sensors. In the future, we will employ the analysis result to design the optimal code matrix for the DCSD fusion rule.

\section{References}

1. Akyildiz, I.F., Su, W., Sankarasubramaniam, Y., Cayirci, E.: Wireless sensor networks: a survey. IEEE Commun. Mag. 38, 102-114 (2002)

2. Aldosari, S.A., Moura, J.M.F.: Detection in decentralized sensor networks. In: Proc. ICASSP 2004, Montreal, Canada (2004)

3. Meyer, G.G.L., Weinert, H.L.: On the design of fault-tolerant signal detectors. IEEE Trans. Acoust. Speech, Signal Processing 34(4), 973-978 (1986)

4. Reibman, A.R., Nolte, L.W.: Optimal fault-tolerant signal detection. IEEE Trans. Acoust. Speech, Signal Processing 38(1), 179-180 (1990)

5. Wang, T.Y., Han, Y.S., Varshney, P.K., Chen, P.N.: Distributed fault-tolerant classification in wireless sensor networks. IEEE J. Select. Areas Commun. 23(4), 724-734 (2005)

6. Varshney, P.K.: Distributed Detection and Data Fusion. Springer, New York (1997)

7. MacWilliams, F.J., Sloane, N.J.A.: The Theory of Error-Correcting Codes. Elsevier, New York (1977)

8. Yao, C., Chen, P.N., Wang, T.Y., Han, Y.S., Varshney, P.K.: Performance analysis and code design for minimum hamming distance fusion in wireless sensor networks. IEEE Trans. Inform. Theory 53(5), 1716-1734 (2007)

9. Chen, P.N., Wang, T.Y., Han, Y.S., Wang, Y.T.: On the design of soft-decision fusion rule for coding approach in wireless sensor networks. In: Int. Conf. on Algorithms, Systems, and Applications, Xian, P. R. China, pp. 140-150 (2006)

10. Wang, T.Y., Han, Y.S., Chen, B., Varshney, P.K.: A combined decision fusion and channel coding scheme for distributed fault-tolerant classification in wireless sensor networks. IEEE Trans. Wireless Commun. 5(7), 1695-1705 (2006)

11. Pai, H.T., Han, Y.S., Sung, J.T.: Two-dimensional coded classification schemes in wireless sensor networks. IEEE Trans. Wireless Commun. (submitted) 